\title{
O Impacto do Mobbing sobre o estresse no trabalho
}

\section{The Impact of Mobbing on stress at work}

\section{El Impacto de Mobbing sobre el estrés en el trabajo}

\author{
Tássia Donadello Ferreira Lima* \\ Universidade Federal Rural do Rio de Janeiro - UFRRJ, Seropédica, Rio de Janeiro, \\ Brasil
}

\section{Marcos Aguiar de Souza**}

Universidade Federal do Rio de Janeiro - UFRJ, Rio de Janeiro, Rio de Janeiro, Brasil

\begin{abstract}
RESUMO
Definido como um conjunto de ações hostis e antiéticas, desenvolvidas no ambiente de trabalho de forma sistemática e recorrente que deixa 0 trabalhador em uma posição indefesa, o mobbing tem sido investigado na sua relação com a saúde e qualidade de vida do trabalhador. Neste sentido, o objetivo no presente trabalho foi (1) o desenvolvimento de três instrumentos para mensuração do mobbing, considerando sua prática por chefes, colegas e subordinados e (2) investigar o impacto do mobbing sobre o estresse no trabalho. Participaram do estudo 300 servidores de uma universidade federal do estado do Rio de Janeiro, sendo 157 homens e 143 mulheres. Os participantes preencheram os três instrumentos para mensuração do mobbing e a EET (Escala de Estresse no Trabalho). A análise fatorial exploratória dos principais eixos com rotação promax revelou uma estrutura ideal de dois fatores em cada instrumento, sendo nomeados de (1) comportamento respeitoso e (2) mobbing praticado por chefes, colegas ou subordinados, conforme o caso. A Modelagem por Equações Estruturais revelou um impacto positivo significativo do mobbing sobre o estresse no trabalho. Da mesma maneira, o comportamento respeitoso por parte de chefes, colegas e subordinados teve influência negativa significativa sobre o estresse no trabalho.
\end{abstract}

Palavras-chave: mobbing, comportamento respeitoso no trabalho, estresse no trabalho.

\begin{abstract}
Defined as a set of hostile and unethical actions developed in the work environment in a systematic and recurring basis which leaves the employee in a defenseless position, mobbing has been investigated in its relation to health and quality of life of workers. In this sense, the goal of this was (1) the development of three instruments for measuring mobbing, considering their practice by bosses, pairs and subordinates, and (2) investigate the impact of mobbing on stress at work. Study participants were 300 servers of a federal university in the state of Rio de Janeiro, with 157 men and 143 women. Participants completed three instruments for measuring the mobbing and the Scale of Work Stress. Exploratory factor analysis with
\end{abstract}


principal axis Promax rotation revealed an ideal two-factor structure for each instrument, being named (1) respectful behavior and (2) mobbing practiced by bosses, colleagues or subordinates. The Structural Equation Modeling revealed a significant positive impact of mobbing on stress at work. Likewise, respectful behavior by bosses, colleagues and subordinates had significant negative influence on job stress.

Keywords: mobbing, respectful behavior at work, stress at work.

\begin{abstract}
RESUMEN
Definido como un conjunto de acciones hostiles y poco éticas desarrolladas en el entorno de trabajo en una forma sistemática y recurrente, que deja al trabajador en estado de indefensión, el mobbing se ha investigado en su relación con la salud y la calidad de vida de los trabajadores. En este sentido, el objetivo de este estúdio fue (1) el desarrollo de tres instrumentos para medir el mobbing, considerando su práctica por jefes, compañeros y subordinados, y (2) investigar el impacto de mobbing sobre el estrés en el trabajo. Los participantes del estudio fueron 300 servidores de una universidad federal en el estado de Río de Janeiro, con 157 hombres y 143 mujeres. Los participantes completaron tres instrumentos para medir el mobbing y la Escala de Estrés Laboral. El análisis factorial exploratorio de ejes principales con rotación Promax reveló una estructura ideal de dos factores para cada instrumento, de ser nombrado (1) el comportamiento respetuoso y (2) el mobbing practicada por los jefes, compañeros 0 subordinados. El Modelos de Ecuaciones Estructurales reveló un impacto positivo significativo del mobbing sobre el estrés en el trabajo. Del mismo modo, un comportamiento respetuoso por jefes, colegas y subordinados tuvo influencia negativa significativa en el estrés laboral.
\end{abstract}

Palabras clave: mobbing, comportamiento respetuoso en el trabajo, estrés en el trabajo.

\title{
1 Introdução
}

As diferentes formas de opressão a que os trabalhadores estão sujeitos nas organizações têm sido amplamente discutidas na literatura por décadas. Tais constatações têm proporcionado 0 desenvolvimento em diferentes países de leis que contribuam para uma relação mais justa do indivíduo com seu trabalho. Em geral havia indicadores de fácil identificação no ambiente de trabalho que permitiam identificar o problema.

Atualmente, muitas das formas diretas de opressão, como o trabalho escravo (Plassat \& Gonçalves, 2005), trabalho infantil (White,1994), discriminação a trabalhadores em função do gênero e de raça (Santos, 2009) que acometiam os trabalhadores estão praticamente controladas, sendo, portanto, de incidência mínima ou mesmo inexistentes em função de esforços inclusive no desenvolvimento de legislação específica. Entretanto, novas formas de opressão têm surgido. É nesse sentido, que se pode falar do mobbing nas organizações. 
O termo mobbing apresenta uma proximidade conceitual com vários termos que têm sido encontrados na literatura. Assim, termos como assédio moral, assédio psicológico, psicoterrorismo e bullying no trabalho, entre outros, têm sido utilizados para representar a situação em que um trabalhador se sente ameaçado por outro trabalhador ou grupo de trabalhadores em seu local de trabalho.

Os estudos sobre mobbing são relativamente recentes, já que há uma dificuldade em identificar e mensurar o fenômeno (Lee \& Brotheridge, 2006). De acordo com Bernal (2010), tal dificuldade se justifica diante da conduta dos assediadores (que buscam se certificar que suas ações não serão facilmente identificadas pelas organizações) e a frequência com que isso ocorre (não é uma ação que se dê de forma constante). Além disso, os perpetradores podem aparecer como trabalhadores úteis e cooperativos em diversos contextos.

Investigado em muitos países, o mobbing apresenta diversos significados. De acordo com Guimarães e Rimoli (2006), na Itália é conhecido como molestie psicologiche, na Inglaterra, Irlanda e Austrália como bullying, bossing e harassment. No Japão é conhecido como Murahachibu. Em Portugal, como coação moral. Nos países hispânicos acoso moral, acoso psicológico ou psicoterrorismo. Na França se chama harcelement moral, na Dinamarca e Noruega é conhecido como mobbing e na Finlândia e Suécia como mobbning.

O fenômeno mobbing é considerado como violência psicológica no trabalho. Este termo foi adotado pela Etologia por Konrad Lorenz a fim de referir o assédio em que um grupo de animais de porte pequeno fazia a um animal solitário, maior e mais forte do que eles. Este termo foi aditado mais tarde por Heineman, médico sueco, para explicar a conduta destrutiva que um grupo de crianças tinham em relação a uma criança sozinha no pátio de uma escola. Em 1980, Leymann aplica este conceito para a violência no âmbito organizacional, definindo mobbing como uma contínua e deliberada violência verbal que um trabalhador sofre de outros trabalhadores (Bernal, 2010).

De acordo com Westhues (2003) o mobbing pode ser definido como uma forma extrema de bullying grupal, na qual o trabalhador é ameaçado por um ou mais funcionários de forma secreta. O objetivo final é isolar e eliminar a vítima. Assim, normalmente são utilizadas formas dissimuladas de boato, fofoca e insinuações que visam desacreditar e desmoralizar o indivíduo em seu trabalho, até que ele se sinta forçado a deixar o emprego (Westhues, 2003, Slallcross, Shejan, \& Ramsay, 2008). De acordo com Leymann (1990), na Suécia em torno da década de 80 do século passado, foram percebidos problemas significativos nos locais de trabalho. Os trabalhadores agrupavam-se para submeter os colegas de trabalho a situações de violência. 
Ao considerarem especificamente o contexto do setor público, Shallcross, Sheehan e Ramsay (2008) concluíram que aqueles que são diferentes do grupo dominante parecem ter um elevado risco de ser um alvo de mobbing, uma vez que a cultura das organizações públicas tende a encorajar a conformidade ao invés de valorizar a diferença. Os autores também concluem que as mulheres são particular risco de se tornarem perpetradoras ou alvos, especialmente naquelas organizações dominadas por mulheres, uma vez que, em levantamento realizado por Namie e Namie (1999) nos Estados Unidos, com 1335 participantes, 84\% dos relatos de mobbing relatavam abusos de mulheres contra outras mulheres.

As estratégias para submeter a vítima ao assédio psicológico são muito variadas. Fidalgo e Piñuel (2004) destacam alguns comportamentos que têm sido identificados nas organizações, como (1) gritar, xingar ou insultar a vítima quando ela está sozinha ou na presença de outras pessoas; (2) atribuir projetos com prazos ou metas que são inatingíveis ou impossíveis de cumprir ou mesmo tarefas intermináveis; (3) retirar responsabilidades, oferecer mudança de rotina com a inclusão de atividades sem importância e não deixar nenhum trabalho para executar; (4) ignorar ou excluir, conversar com uma terceira pessoa, insinuando a inexistência, seja no escritório ou em reuniões que frequenta, tratar a pessoa como se fosse invisível; (5) reter informações cruciais para o seu trabalho ou manipular para induzir ao erro em seu desempenho no trabalho, e depois acusá-lo de negligência ou má conduta profissional; (6) espalhar pela empresa rumores maliciosos e calúnias que prejudicam sua reputação, sua imagem ou o seu profissionalismo; (7) subestimar, não valorizar ou não reconhecer o esforço realizado em algum trabalho; (8) ignorar o sucesso profissional, atribuindo maliciosamente a outras pessoas, ou elementos externos como: causalidade, sorte, e condições de mercado, entre outros; (9) criticar continuamente o seu trabalho, suas ideias, propostas e soluções; (10) punir severamente qualquer tomada de decisão ou iniciativa pessoal no desempenho do trabalho; (11) ridicularizar o seu trabalho, as suas ideias ou resultados para outros trabalhadores; (12) incentivar os outros trabalhadores a participar em qualquer uma dessas ações anteriores de persuasão, coerção e abuso de autoridade e (13) invadir a privacidade, intervir no seu e-mail, telefone, verificar seus documentos, armários, e gavetas, de forma maliciosa e subtrair elementos importantes para 0 seu trabalho. Todos esses comportamentos resultam em problemas no bem estar, na saúde física e psicológica da vítima.

Leymann (1990) apresentou quatro fases em que o mobbing pode ocorrer. A primeira fase do mobbing é definida como a fase de conflito. Nesta fase, os conflitos pessoais, considerados normais entre pessoas com diferentes objetivos e interesses apresentam problemas 
que poderiam ser resolvidos de uma forma positiva. Entretanto, quando esses assuntos não são esclarecidos da melhor forma as relações tornam-se inflexíveis e a partir daí surgem os problemas a serem enfrentados.

A segunda fase do mobbing é conhecida como estigmatização. É quando o assediador humilha, ridiculariza e isola a vítima no seu convívio social. Seu comportamento é perverso. Esta é a fase em que o mobbing ocorre definitivamente, podendo ser uma fase de longa duração, já que pode ocorrer a estigmatização dessa agressão por parte das pessoas que convivem com a vítima (Guimarães \& Rimoli, 2006).

A terceira fase do mobbing é entendida como uma fase em que a organização na qual se encontra a vítima utiliza estratégias de intervenção. É quando a organização toma ciência dos conflitos. A solução para estes conflitos pode ser positiva ou negativa. A solução é positiva quando o trabalhador ou o agressor é transferido do seu local de trabalho, ou quando um trabalho é realizado para que as humilhações não voltem a ocorrer, conscientizando assim os demais funcionários e quando há uma punição para o agressor. A solução é negativa quando a investigação dos conflitos se torna escassa e a vítima é considerada um problema que precisa ser combatido, a organização destaca as características distorcidas e errôneas da vítima sem perceber que a origem do problema está no agressor, sendo assim, a organização também pode ser considerada como aquela que colabora ativa e passivamente para o mobbing no ambiente de trabalho (Guimarães \& Rimoli, 2006).

$\mathrm{Na}$ quarta fase do mobbing, a fase da marginalização, ocorre a exclusão do trabalhador da sua vida laboral. Esta fase irá ocorrer quando a intervenção na situação de mobbing não é adequadamente resolvida (fase 3 ). Neste momento, a vítima abandona seu emprego, muitas vezes depois de muitos períodos de licença. Há ainda trabalhadores de instituições públicas que solicitam mudança do seu local de trabalho, em organizações privadas, isso é mais difícil de ser aceito, no entanto, o trabalhador permanece no mesmo posto de trabalho sofrendo consequências graves de saúde e em casos extremos, pode-se chegar ao suicídio.

Para Shallcross, Sheehan e Ramsay (2008), outra forma de analisar o mobbing a partir das fases tem sido utilizada em diferentes estudos. Nessa outra concepção, existiriam cinco fases distintas: (1) conflito inicial ou fase de incidente crítico; (2) Maior direcionamento do mobbing para o alvo. (3) Ocorre a intervenção da organização, que via de regra termina por aumentar os níveis de dano. (4) O alvo de mobbing é acusado de ser uma pessoa difícil ou com problemas mentais. (5) o alvo estimulado a sair do emprego ou mesmo demitido. 
A dificuldade de identificação do mobbing se soma a dificuldade de mensurá-lo. Existem poucos instrumentos disponíveis e os estudos, em muitos casos, não permitem ainda concluir pela real contribuição dos instrumentos para um avanço na área. Abaixo são apresentados três instrumentos disponíveis para investigação do fenômeno: 0 barômetro CISNEROS (Fidalgo \& Piñuel, 2004), o LIPT-60 (Leymann Inventory of Psychological Terrorization) e o NAQ (Negative Acts Questionnaire - Questionário de Atos Negativos - Einarsen \& Hoel, 2001). Tais instrumentos serviram de base para desenvolvimento dos itens do instrumento proposto no presente estudo.

O barômetro Cisneros, desenvolvido por Fidalgo e Piñuel (2004) é composto por 43 itens destinados a avaliar o mobbing cometido por chefes, colegas e subordinados. Em cada item, além de assinalar 0 praticante do mobbing, o respondente deve indicar a frequência que tal comportamento ocorre, variando desde 0 (nunca) a 6 (todos os dias).

O LIPT-60 (Leymann Inventory of Psychological Terrorization Inventário Leymann de Terror Psicológico), desenvolvido por Leymann e Tallgren (1989), é composto por 60 itens divididos em 6 fatores: Desprestígio Laboral (14 itens), Entorpecimento do Progresso (7 itens), Bloqueio da Comunicação (9 itens), Intimidação Encoberta (7 itens), Intimidação Manifesta (6 itens) e Desprestígio Pessoal (7 itens). Além dos itens que constituem os fatores, o LIPT possui ainda 10 itens que não são enquadrados em fatores específicos. 0 respondente classifica em uma escala de 0 a 4 a intensidade de ocorrência dos comportamentos descritos pelos itens.

O NAQ é composto de uma lista de 21 atos negativos relacionados tanto a exposição a comportamentos específicos de bullying quanto a sentimentos de vitimização no trabalho. É pedido ao respondente que indique a frequência com que tem estado sujeito aos atos negativos listados nos últimos seis meses.

De acordo com Guimarães e Rimoli (2006), o mobbing está fortemente associado ao estresse no trabalho, motivo pelo qual diversos autores têm evidenciado o conjunto de prejuízos à saúde e qualidade de vida do trabalhador associado ao mobbing, podendo este ser considerado um estressor social grave. É nesse sentido que, visando obter evidências da validade externa dos instrumentos propostos no presente estudo, surgiu o interesse de investigar 0 impacto do mobbing sobre o estresse no trabalho.

Para Schabracq (2003) o estresse é um antigo padrão de reação animal que nos permite a adaptação às circunstâncias que ameaçam a vida e que não podemos lidar com os nossos padrões habituais de comportamento. Assim, deve ser compreendido que o estresse pode estar presente em várias circunstâncias da vida, sendo o trabalho apenas um dentre os diversos contextos em que o indivíduo pode vivenciar o estresse (Cox, 1993). 
De acordo com Selye (1950), o padrão de reação ao estresse é basicamente o mesmo, independentemente da fonte estressora. É nesse sentido, que define o estresse como uma síndrome geral de adaptação. Tudo que provoca estresse põe em perigo a vida, a menos que sejam apresentadas respostas adaptativas adequadas; inversamente, qualquer coisa que coloca em risco a vida provoca estresse e respostas adaptativas. Assim, a adaptabilidade e a resistência ao estresse são requisitos fundamentais para a vida.

O estresse no trabalho é uma condição ou situação que requer uma resposta adaptativa por parte do empregado. Ambiguidade de papel e conflito de papel são os estressores mais citados nas pesquisas sobre estresse ocupacional. A ambiguidade de papel é a extensão em que os empregados estão incertos sobre quais são suas funções e responsabilidades na organização. O conflito de papel surge quando as pessoas experienciam demandas incompatíveis no trabalho ou entre atividades dentro e fora do trabalho (Tang, 2008).

Para Jex (2002), apesar do pioneirismo de Selye nos estudos sobre o estresse em geral, as primeiras investigações sobre o estresse ocupacional são atribuídas a Walter Cannon, que no início do século passado investigou o relacionamento entre emoções e respostas fisiológicas, criando o conceito de homeostase como o esforço do organismo para restaurar o funcionamento fisiológico normal quando algum desvio ocorre. Assim, as condições estressoras no trabalho podem ser definidas como eventos aversivos que requerem uma resposta adaptativa por parte do empregado para retornar ao funcionamento normal.

Segundo Cox (1993), o estresse no trabalho tem sido definido a partir de três principais abordagens. A primeira define o estresse no trabalho como uma característica nociva ou aversiva do ambiente de trabalho, sendo o mesmo considerado como uma variável independente - a causa ambiental do problema de saúde. Na segunda abordagem o estresse no trabalho é considerado como uma variável dependente, sendo destacados os efeitos fisiológicos advindos da exposição do indivíduo a um ambiente prejudicial e ameaçador. Finalmente, na terceira abordagem 0 estresse no trabalho é definido em termos de uma interação dinâmica entre a pessoa e seu ambiente de trabalho.

Paschoal e Tamayo (2004) definem o estresse ocupacional como um processo em que o indivíduo percebe as demandas do trabalho como fatores estressores que excedem sua capacidade de enfrentamento, provocando reações negativas.

Cartwright e Whatmore (2005) consideram que o estresse tem sido identificado como um fator importante em problemas de saúde, especialmente a saúde psicológica. Em contrapartida, Heaphy e Dutton (2008) defendem que interações sociais positivas no ambiente 
de trabalho proporcionam efeitos fisiológicos benéficos para os trabalhadores.

Os prejuízos advindos do estresse ocupacional para os trabalhadores e para as organizações têm sido amplamente documentados (Cartwright e Whatmore, 2005; Paschoal \& Tamayo, 2004; Jex, 2002; Cox,1993). Jong (2002) considera que o estresse envolve estados emocionais negativos como ansiedade, raiva e depressão que surgem em função do desequilíbrio entre o impacto dos estressores e a capacidade do indivíduo em lidar com eles. Apesar das inúmeras divergências existentes entre os pesquisadores em relação ao conceito de estresse no trabalho, existe um consenso, para que um evento possa ser considerado como estressor: é necessário que o indivíduo assim o classifique. Nesse sentido, a percepção individual funciona como um mediador do impacto do ambiente de trabalho sobre o trabalhador (Paschoal \& Tamayo, 2004).

Paschoal e Tamayo (2005) consideram que muitos estudos têm sido desenvolvidos buscando identificar variáveis capazes de influenciar o estresse no trabalho. De fato, o estresse tem sido investigado na sua relação com valores laborais (Paschoal \& Tamayo, 2005), conflito família-trabalho (Paschoal \& Tamayo, 2005), valores organizacionais (Canova \& Porto, 2010), qualidade de vida (Sadir, Bignotto \& Lipp, 2010), atenção concentrada (Baptista, Rueda \& Sisto, 2007), prática de atividade física (Tamayo, 2001) entre outras variáveis. No presente estudo, o interesse é a investigação do estresse no trabalho como variável dependente, tendo como o mobbing o estressor organizacional.

\section{Método}

\subsection{Participantes}

Participaram do estudo 300 servidores da Universidade Federal Rural do Rio de Janeiro, sendo 157 do sexo masculino e 143 do sexo feminino, com idade variando de 21 a 68 anos (média de 44,39 anos e desvio padrão de 12, 03 anos). O tempo de serviço médio foi de 14,96 anos (desvio padrão de 13 anos), variando de 1 a 45 anos de serviço. Dos participantes, 90 (30\%) eram solteiros, $171(57 \%)$ casados e $39(13 \%)$ enquadrados em outras situações (divorciados e viúvos, entre outros casos apontados).

\subsection{Instrumentos}

Para a coleta de dados do estudo foi utilizado um questionário composto de 3 partes. A primeira teve como objetivo a coleta de 
dados sócio-demográficos: sexo, idade, estado civil, tempo de serviço na universidade.

A segunda parte foi constituída pela Escala de Mobbing, objeto do presente estudo. A escala de mobbing foi elaborada com base no LIPT, adaptado por Rivera e Rodríguez (2005); na escala Barómetro CISNEROS e no Questionário de Ações Negativas (NAQ). Os itens de tais instrumentos foram reunidos em um instrumento único, que foi aplicado a um total de 10 servidores, que indicaram (1) quais situações descritas já tinham sido observadas no contexto da universidade e (2) o que entendiam em relação ao que estava sendo descrito em cada item. Ao final de tal processo, o instrumento passou a contar com 26 itens. Os itens deveriam ser avaliados em uma gradação de 5 pontos, variando desde nunca (1) nunca até (5) sempre. Na escala o respondente deveria ainda indicar os autores envolvidos, podendo ser: chefe imediato, companheiros de trabalho e subordinados. A previsão é que seriam obtidos 3 instrumentos independentes, de modo a mensurar o mobbing praticado por chefes, colegas e subordinados.

$\mathrm{Na}$ forma como foi elaborada a escala é destinada a mensurar o mobbing de forma unidimensional, porém, a partir de 3 escalas independentes. $O$ instrumento inicial constou de 26 itens, que foram considerados adequados para mensurar o fenômeno no contexto da IFES investigada. Assim, considerou-se a Escala Brasileira de Mobbing - Chefe, Escala Brasileira de Mobbing - Companheiros de Trabalho e a Escala Brasileira de Mobbing - Subordinados. Os fatores denominados nesta escala se referem aos autores do mobbing. Cada escala apresentou índices satisfatórios em relação a fatorabilidade dos itens (KMO e Teste de Esfericidade de Bartlett, à porcentagem de variância total explicada e ao coeficiente alfa de Cronbach).

A terceira parte do instrumento constou da Escala de Estresse no Trabalho - EET (Paschoal e Tamayo, 2004). Tal instrumento é composto por 26 itens que juntos se destinam a mensurar situações causadoras de estresse no ambiente e trabalho. No presente estudo a EET foi replicada a estrutura unifatorial do instrumento, que apresentou índice de consistência interna (alfa de Cronbach) de 0,933 sendo explicado $41,62 \%$ da variância observada. Tais resultados são ainda mais satisfatórios que os obtidos pelos autores. São itens típicos da EET "Fico de mau humor por me sentir isolado na organização" e "a falta de autonomia na execução do meu trabalho tem sido desgastante".

\subsection{Procedimentos éticos}

Inicialmente foi discutido com os setores de trabalho na UFRRJ o objetivo geral do estudo, assim como a relevância do mesmo, garantindo o anonimato, o sigilo absoluto das respostas dos 
participantes e o compromisso de apresentação dos resultados obtidos. Foi explicado também aos servidores que o estudo havia sido aprovado pelo comitê de ética em pesquisa da universidade. A escala foi aplicada individualmente, com consentimento dos participantes que aceitaram voluntariamente participar do estudo. A pesquisa foi aprovada pelo Comitê de Ética segundo a Resolução 196/96 da CONEP (Comissão Nacional de Ética em Pesquisas de 1996), de acordo com o parecer $\mathrm{n}^{0 .}$ 298/2012, de 01/02/2013.

\section{Resultados}

Os procedimentos de validação da Escala de Mobbing envolveram a análise fatorial exploratória e o cálculo da consistência interna com a utilização do coeficiente alfa de Cronbach. O programa estatístico que utilizado foi o software SPSS 19 (Statiscal Package for the Social Sciences) e o AMOS (Analysis of Moment Structures), ambos com licença adquirida pelo Departamento de Psicologia da UFRRJ, para a análise quantitativa dos dados. Na análise fatorial exploratória, foram retidos em cada fator os itens que apresentaram carga fatorial igual ou superior a 0,30 .

Considerando-se inicialmente o mobbing praticado por chefes, os 26 itens foram submetidos a uma Análise Fatorial Exploratória (AFE) dos principais eixos e com rotação promax. Assim, o KMO obtido foi de 0,852 e o teste de esfericidade de Bartlett foi significativo ao nível de 0,001 , indicando a adequação de realização dos procedimentos de AFE nos itens relativo ao comportamento dos colegas no trabalho. A melhor estrutura obtida (menor número de fatores e máxima variância explicada) foi a de dois fatores que juntos explicaram $49,78 \%$ da variância. O coeficiente alfa de Cronbach obtido foi de 0,79 para o Fator 1 (comportamento respeitoso do chefe/supervisor) e 0,73 para o fator 2 (mobbing perpetrado pelo chefe/supervisor). Os itens que permaneceram na escala são descritos na abaixo. 
Tabela 1

Itens por fator e respectivas cargas fatoriais da Escala de Comportamento do Chefe

\begin{tabular}{|c|c|c|}
\hline \multirow[b]{2}{*}{ Item } & \multicolumn{2}{|c|}{ Carga Fatorial } \\
\hline & Fator 1 & Fator 2 \\
\hline $\begin{array}{l}\text { 3. Valoriza o meu esforço profissional e o trabalho } \\
\text { que executo. }\end{array}$ & 0,74 & \\
\hline $\begin{array}{l}\text { 5. Ordena-me tarefas de acordo com as minhas } \\
\text { competências. }\end{array}$ & 0,68 & \\
\hline $\begin{array}{l}\text { 6. Dá ordens coerentes e claras para o meu } \\
\text { trabalho. }\end{array}$ & 0,66 & \\
\hline $\begin{array}{l}\text { 9. Oferece-me condições adequadas para o } \\
\text { cumprimento das tarefas. }\end{array}$ & 0,59 & \\
\hline 23. Faz com que eu me sinta apoiado. & 0,55 & \\
\hline 10. Eleva a voz quando eu falo. & & 0,81 \\
\hline 14. Evita falar comigo ou ficar próximo a mim. & & 0,64 \\
\hline $\begin{array}{l}\text { 16. Distorce o que eu falo ou faço no meu } \\
\text { trabalho. }\end{array}$ & & 0,63 \\
\hline $\begin{array}{l}\text { 22. Faz-me vítima de insultos, humilhações e } \\
\text { desprezos em público ou em frente aos colegas de } \\
\text { trabalho. }\end{array}$ & & 0,57 \\
\hline
\end{tabular}

Os mesmos procedimentos de análise fatorial exploratória foram adotados em relação a escala de comportamento dos colegas. Assim, o KMO obtido foi de 0,731 e o teste de esfericidade de Bartlett significativo ao nível de 0,001 , indicando a adequação de realização dos procedimentos de AFE nos itens relativo ao comportamento dos colegas no trabalho. Foram obtidos dois fatores, que juntos foram responsáveis por $47,33 \%$ da variância observada.

O Fator 1 - Comportamento Respeitoso dos Colegas - foi constituído por 4 itens que diziam respeito a comportamentos éticos e respeitosos por parte dos colegas de trabalho. O coeficiente alfa de Cronbach obtido no fator foi de 0,74. Já o Fator 2 - Mobbing praticado pelos colegas - foi composto por 5 itens que descrevem comportamentos agressivos ou desrespeitosos por partes dos colegas de trabalho. O Coeficiente alfa de Cronbach foi de 0,79. Na Tabela 2 são apresentados os itens distribuídos em cada fator, com as respectivas cargas fatoriais. 
Tabela 2

Itens por fator e respectivas cargas fatoriais da Escala de Comportamento dos Colegas

\begin{tabular}{lcc}
\hline \multicolumn{1}{c}{ Item } & \multicolumn{2}{c}{ Carga Fatorial } \\
& Fator 1 & Fator 2 \\
\hline $\begin{array}{l}\text { 6. Dão ordens coerentes e claras para o meu } \\
\text { trabalho. }\end{array}$ & 0,708 & \\
$\begin{array}{l}\text { 5. Ordenam-me tarefas de acordo com as minhas } \\
\text { competências. }\end{array}$ & 0,698 & \\
$\begin{array}{l}\text { 9. Oferecem-me condições adequadas para o } \\
\text { cumprimento das tarefas. }\end{array}$ & 0,650 & \\
3. Valoriza o meu esforço profissional e o trabalho & 0,517 & \\
que executo. & & 0,596 \\
10. Elevam a voz quando eu falo. & & 0,590 \\
$\begin{array}{l}\text { 22. Fazem-me vítima de insultos, humilhações e } \\
\text { desprezos em público ou em frente aos colegas } \\
\text { de trabalho. }\end{array}$ & & 0,590 \\
$\begin{array}{l}\text { 14. Evitam falar comigo ou ficar próximos a mim. } \\
\text { 13. Interrompem-me de forma pejorativa e/ou } \\
\text { humilhante quando falo. }\end{array}$ & 0,559 \\
$\begin{array}{l}\text { 16. Distorcem o que eu falo ou faço no meu } \\
\text { trabalho. }\end{array}$ & 0,540 \\
\hline
\end{tabular}

Os mesmos procedimentos adotados para a AFE nas duas escalas anteriores foi utilizado para analisar os itens da Escala de Comportamento dos Subordinados. O KMO obtido foi de 0,90 e o teste de esfericidade de Barttlet foi significativo ao nível de 0,001. A exemplo dos outros dois instrumentos, foram obtidos 2 fatores. $O$ primeiro foi nomeado de Comportamento Respeitoso por parte dos Subordinados, sendo composto por 8 itens relativos a comportamentos éticos e respeitosos no ambiente e trabalho. 0 segundo fator foi nomeado de Mobbing Praticado pelos Subordinados, composto por 5 itens relativos a comportamentos agressivos ou desrespeitosos praticados pelos subordinados no ambiente de trabalho. O coeficiente alfa de Cronbach obtido foi, respectivamente, de 0,92 e 0,75 . Juntos os fatores explicaram $51,98 \%$ da variância observada.

Na Tabela 3 são apresentados os itens distribuídos em cada fator, com as respectivas cargas fatoriais. 
Tabela 3

Itens por fator e respectivas cargas fatoriais da Escala de Comportamento dos Subordinados

\begin{tabular}{lcc}
\hline \multicolumn{1}{c}{ Item } & \multicolumn{2}{c}{ Carga Fatorial } \\
& Fator 1 & Fator 2 \\
\hline 24. Tratam-me de forma educada e respeitosa. & 0,845 & \\
23. Fazem com que eu me sinta apoiado. & 0,832 & \\
15. Respeitam os meus valores e ética profissional. & 0,823 & \\
17. Aceitam minhas opiniões e pontos de vista & 0,794 & \\
12. Tornam o local de trabalho um ambiente calmo & 0,753 & \\
e harmonioso. & 0,729 \\
20. Respeitam as minhas crenças religiosas. & 0,723 \\
26. Fazem do meu ambiente de trabalho um lugar & & \\
seguro, sem colocar em risco a minha vida. & & 0,643 \\
3. Valorizam o meu esforço profissional e o trabalho & 0,659 & \\
que executo. & & 0,641 \\
16. Distorcem o que eu falo ou faço no meu & & \\
trabalho. & & 0,588 \\
22. Fazem-me vitima de insultos, humilhações e & & \\
desprezos em público ou em frente aos colegas de & & \\
trabalho. & & \\
10. Elevam a voz quando eu falo. & & \\
8. Fazem controle do meu horário de trabalho de & & \\
forma mais intensa que aos demais. & &
\end{tabular}

Os procedimentos de AFE dos principais eixos com rotação Promax indicaram a adequação dos três instrumentos independentes propostos. Assim dos 26 itens que constavam em cada instrumento, foram retirados 17 na análise do mobbing praticado pelo chefe e pelo colega e apenas 14 na análise do mobbing praticado pelos subordinados. Em seguida foi realizada uma análise descritiva dos dados, conforme pode ser observado na Tabela 4. 
Tabela 4

Média, mediana e desvio padrão dos participantes nas medidas de estresse no trabalho e de mobbing praticado pelos chefes, colegas e subordinados

\begin{tabular}{|c|c|c|c|}
\hline Variável & Média & Mediana & $\begin{array}{l}\text { Desvio } \\
\text { Padrão } \\
\end{array}$ \\
\hline 1. Estresse no Trabalho & 1,97 & 1,91 & 0,70 \\
\hline $\begin{array}{l}\text { 2. Comportamento Respeitoso por parte } \\
\text { do Chefe }\end{array}$ & 3,77 & 3,80 & 0,80 \\
\hline $\begin{array}{l}\text { 3. Comportamento Respeitoso por parte } \\
\text { dos Colegas }\end{array}$ & 3,56 & 3,75 & 0,95 \\
\hline $\begin{array}{l}\text { 4. Comportamento Respeitoso por parte } \\
\text { dos Subordinados }\end{array}$ & 3,68 & 4,00 & 1,16 \\
\hline 5. Mobbing praticado pelo Chefe & 1,47 & 1,25 & 0,69 \\
\hline 6. Mobbing praticado pelos Colegas & 1,40 & 1,20 & 0,57 \\
\hline 7. Mobbing praticado pelos Subordinados & 1,37 & 1,00 & 0,61 \\
\hline
\end{tabular}

Em relação a média de estresse no trabalho apresentada pelos participantes, é possível afirmar ser relativamente baixo, uma vez que em outros estudos realizados com o mesmo instrumento têm sido obtidas médias mais elevadas (Martinez \& Latorre, 2009; Paschoal \& Tamayo, 2005).

Obtidos os instrumentos para mensuração do comportamento de Chefes, Colegas e Subordinados no trabalho, foi realizada a análise do impacto do mobbing sobre o estresse no trabalho, considerando as três escalas independentes, utilizando a modelagem por equações estruturais. Assim, foram considerados os índices de qualidade do ajustamento do modelo. O método de análise utilizado foi o de máxima verossimilhança, por ser um método tradicional e o mais utilizado (Marôco, 2010). Para análise do modelo foram considerados (1) índices absolutos, (2) índices relativos, (3) índices de parcimônia e (4) índices de discrepância populacional. Tais índices permitem avaliar a adequação do modelo proposto (Hair et al., 2005; Marôco, 2010).

Os índices absolutos avaliam a qualidade do modelo, sem compará-lo com outros modelos possíveis. Um índice que tem sido amplamente considerado é o $\mathrm{X}^{2} / \mathrm{gl}$ (qui-quadrado dividido pelos graus de liberdade). $O$ valor 1 nesse índice indica um ajustamento perfeito. Entretanto, considera-se aceitação um valor de até 5 .

Outros índices absolutos amplamente utilizados são o RMR (Root Mean Square Residual) e o GFI (Goodness of Fit Index). Quanto menor o RMR, melhor é o ajustamento do modelo, sendo 0 o valor de um ajustamento perfeito. O GFI de um modelo perfeito teria valor 1 . Entretanto, considera-se um bom ajustamento quando seu valor é acima de 0,9 . 
Os índices relativos avaliam a qualidade do modelo que está sendo testado em relação a dois modelos hipotéticos: um modelo de pior ajustamento possível e um modelo de melhor ajustamento possível. Alguns dos critérios de análise que se enquadram nesta categoria são o NFI e o RFI.

O NFI (Normed Fit Index) de um modelo perfeito ou ideal possui valor 1. Entretanto, valores acima de 0,9 são considerados aceitáveis. No presente estudo o valor obtido foi de 0,91 . Em relação ao RFI (Relative Fit Index), valores acima de 0.9 são considerados aceitáveis, sendo 1 o valor ideal.

Os índices de parcimônia têm como objetivo compensar o melhor ajuste que modelos mais complexos podem apresentar. Alguns dos índices de parcimônia utilizados em estudos de modelagem por equações estruturais são PNFI (Parcimony Normed Fit Index) e PGFI (Parsimony Goodness of Fit Index). Valores superiores a 0,5 são indicativos de um modelo bem ajustado.

Os índices de discrepância populacional comparam o modelo obtido com os participantes do estudo com aquele que seria obtido considerando toda a população. Um índice que é amplamente utilizado é o RMSEA (Root Mean Error of Approximation). O modelo é considerado inapropriado quando o valor de RMSEA é superior a 0,10, sendo muito bom quando o valor é inferior a 0,05. Contudo, o valor do RMSEA tende a aumentar conforme aumenta o número de variáveis no modelo, o que indica a necessidade de uma maior reflexão na utilização deste índice.

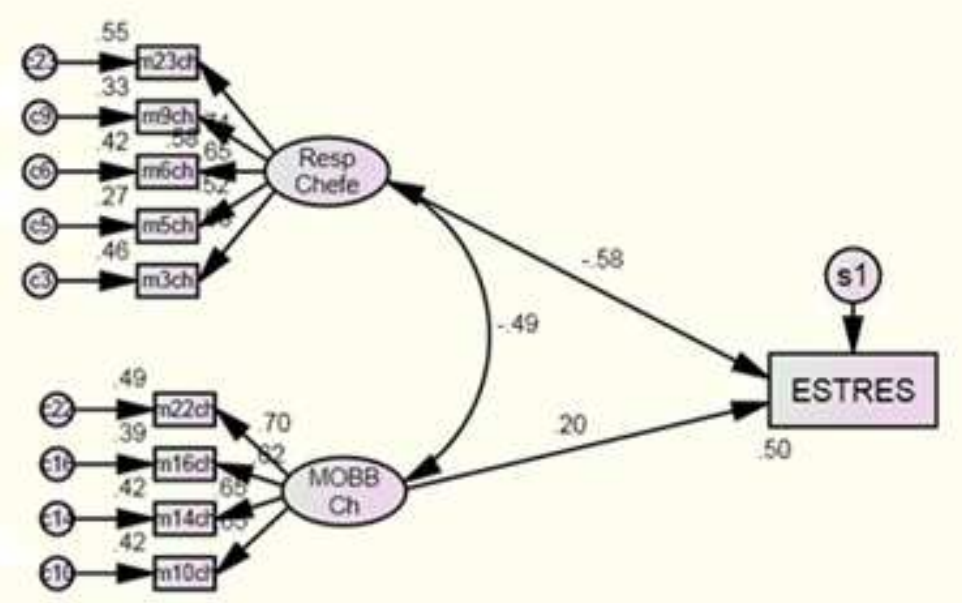

Figura 1- Modelo do impacto do comportamento do chefe sobre o estresse no trabalho. 
Tabela 5

Indices de ajuste do modelo de impacto do comportamento do chefe sobre o estresse no trabalho

\begin{tabular}{ccc}
\hline Índices & $\begin{array}{c}\text { Valor } \\
\text { obtido }\end{array}$ & $\begin{array}{c}\text { Índice } \\
\text { satisfatório? }\end{array}$ \\
\hline X2/gl & 2,19 & Sim \\
GFI & 0,96 & Sim \\
RMR & 0,05 & Sim \\
RFI & 0,91 & Sim \\
CFI & 0,96 & Sim \\
PCFI & 0,66 & Sim \\
PNFI & 0,64 & Sim \\
RMSEA & 0,06 & Sim \\
\hline
\end{tabular}

Como pode ser observado na figura 1 e na Tabela 4, foram obtidas evidências satisfatórias do modelo proposto, relativo ao impacto do comportamento do chefe (comportamento de respeito ou de mobbing) sobre o estresse no trabalho.

O comportamento de respeito de chefe, como hipotetizado, teve um impacto negativo significativo sobre $o$ estresse no trabalho ( $p<$ 0,000 ). Também como esperado, o mobbing praticado pelo chefe teve um impacto positivo sobre o estresse no trabalho $(p<0,01)$. Observa-se também na figura uma correlação negativa significativa entre a medida do mobbing praticado pelo chefe e o comportamento respeitoso praticado pelo chefe $(p<0,000)$.

Em seguida, foi analisado o modelo do comportamento dos colegas (comportamento respeitoso e mobbing) sobre o estresse no trabalho.

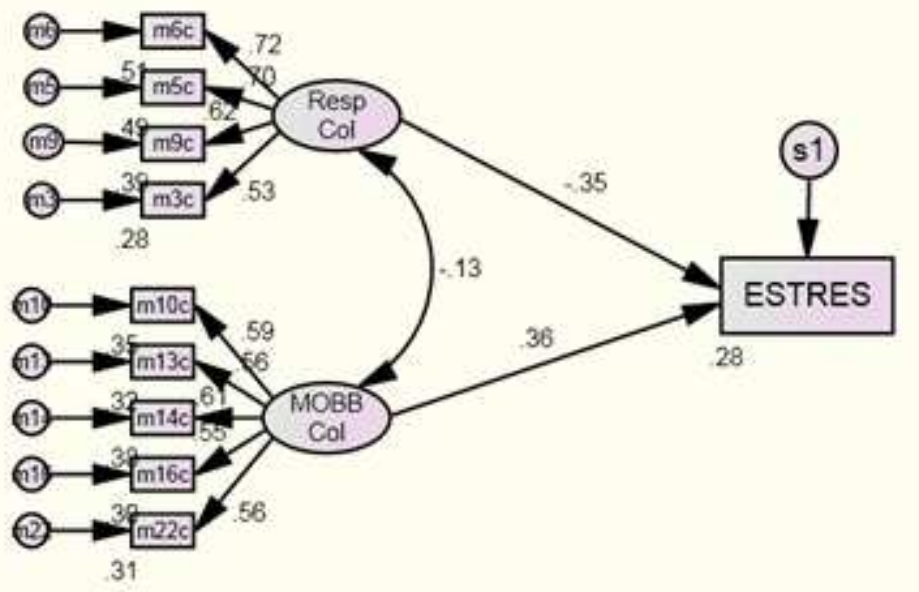

Figura 2 - Modelo do impacto do comportamento dos colegas sobre o estresse no trabalho. 
Tabela 6

Indices de ajuste do modelo de impacto do comportamento dos colegas sobre o estresse no trabalho

\begin{tabular}{ccc}
\hline Índices & Valor obtido & $\begin{array}{c}\text { Índice } \\
\text { satisfatório? }\end{array}$ \\
\hline X2/gl & 1,87 & Sim \\
GFI & 0,96 & Sim \\
RMR & 0,04 & Sim \\
RFI & 0,86 & Sim \\
CFI & 0,95 & Sim \\
PCFI & 0,70 & Sim \\
PNFI & 0,66 & Sim \\
RMSEA & $0,05(0,03-$ & Sim \\
& $0,07)$ & \\
\hline
\end{tabular}

Como esperado, os resultados evidenciaram que o comportamento respeitoso por parte dos colegas tem um impacto negativo significativo $(p<0,000)$ sobre o estresse no trabalho. Da mesma forma, foi significativo também o impacto positivo do mobbing praticado pelos colegas sobre o estresse no trabalho ( $p<0,000$ ). A correlação entre o comportamento respeitoso e o mobbing praticado pelos colegas, como esperado, foi negativa e significativa ( $p<$ $0,000)$.

Finalmente, foi avaliado o impacto do comportamento dos subordinados (comportamento respeitoso e de mobbing) sobre 0 estresse no trabalho. Mais uma vez foi utilizada a análise por equações estruturais, sendo obtido o modelo descrito na figura 3 .

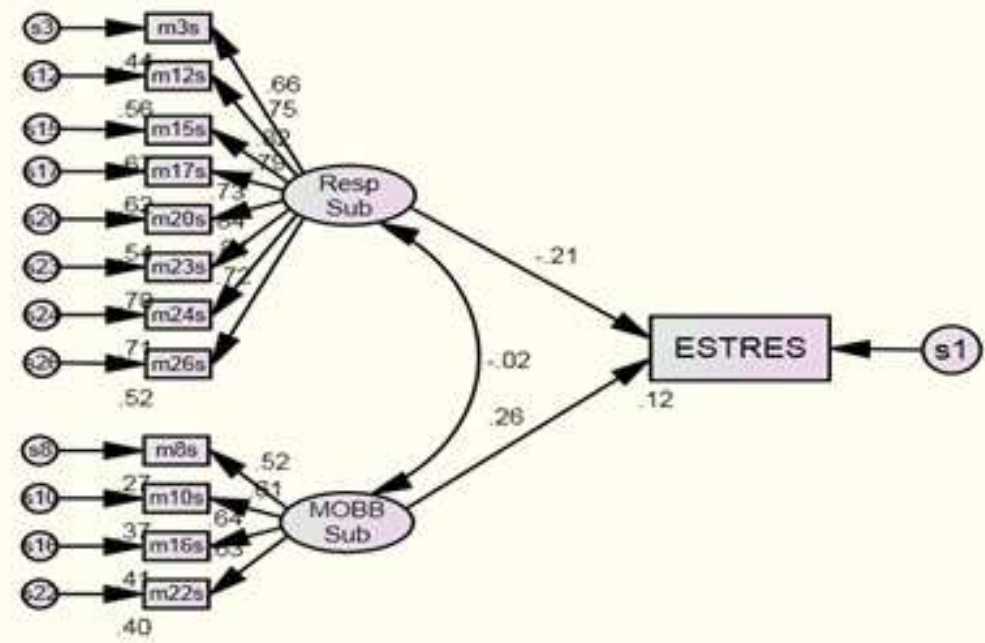

Figura 3 - Modelo do impacto do comportamento dos subordinados sobre o estresse no trabalho. 
Tabela 7

Indices de ajuste do modelo de impacto do comportamento dos subordinados sobre o estresse no trabalho

\begin{tabular}{ccc}
\hline Índices & Valor obtido & $\begin{array}{c}\text { Índice } \\
\text { satisfatório? }\end{array}$ \\
\hline X2/gl & 1,95 & Sim \\
GFI & 0,94 & Sim \\
RMR & 0,06 & Sim \\
RFI & 0,91 & Sim \\
CFI & 0,96 & Sim \\
PCFI & 0,78 & Sim \\
PNFI & 0,75 & Sim \\
RMSEA & $0,06(0,04-$ & Sim \\
& $0,07)$ & \\
\hline
\end{tabular}

O modelo de impacto do comportamento dos subordinados sobre o estresse no trabalho apresentou como esperado um resultado significativo tanto do impacto negativo do comportamento respeitoso, como do mobbing praticado pelos subordinados (em ambos os casos, $\mathrm{p}<0,000)$. A correlação entre os dois tipos de comportamento dos subordinados, entretanto, não foi significativa.

Tomados em conjunto, os resultados permitem concluir pelo impacto negativo que o mobbing tem no ambiente de trabalho, tendo em vista o aumento de níveis de estresse no trabalho que sua presença ocasiona.

Os resultados indicam que o mobbing praticado pelos colegas teve um maior impacto para o desenvolvimento do estresse ocupacional, seguido pelo mobbing praticado pelo chefe e estando em último lugar o mobbing praticado pelos subordinados. Tal resultado parece lógico ao se considerar a maior proximidade que o trabalhador tem com seus pares, servindo inclusive de modelo comparativo para a avaliação de diferentes situações e procedimentos dentro da organização. Também é entre os pares que o trabalhador espera ter maior possibilidade de interação social, reduzindo as incertezas do ambiente de trabalho.

Houve o interesse em comparar as variáveis do estudo em grupos constituídos em função do sexo e do estado civil. Entretanto, a utilização do teste $t$ de Student não revelou diferenças significativas entre homens e mulheres ou entre casados e solteiros. A inexistência de diferenças significativas entre homens e mulheres no contexto organizacional vem sendo constatada em diferentes estudos. De fato, em um contexto em que há ampla discussão sobre a igualdade entre os sexos, inclusive garantida por lei, é de se esperar que práticas mais igualitárias tomem lugar.

Adicionalmente, foi realizado um cálculo correlacional entre as variáveis do estudo, como apresentado na Tabela 8. 
Tabela 8

Calculo correlacional entre as variáveis do estudo com a utilização do coeficiente de correlação linear de Pearson

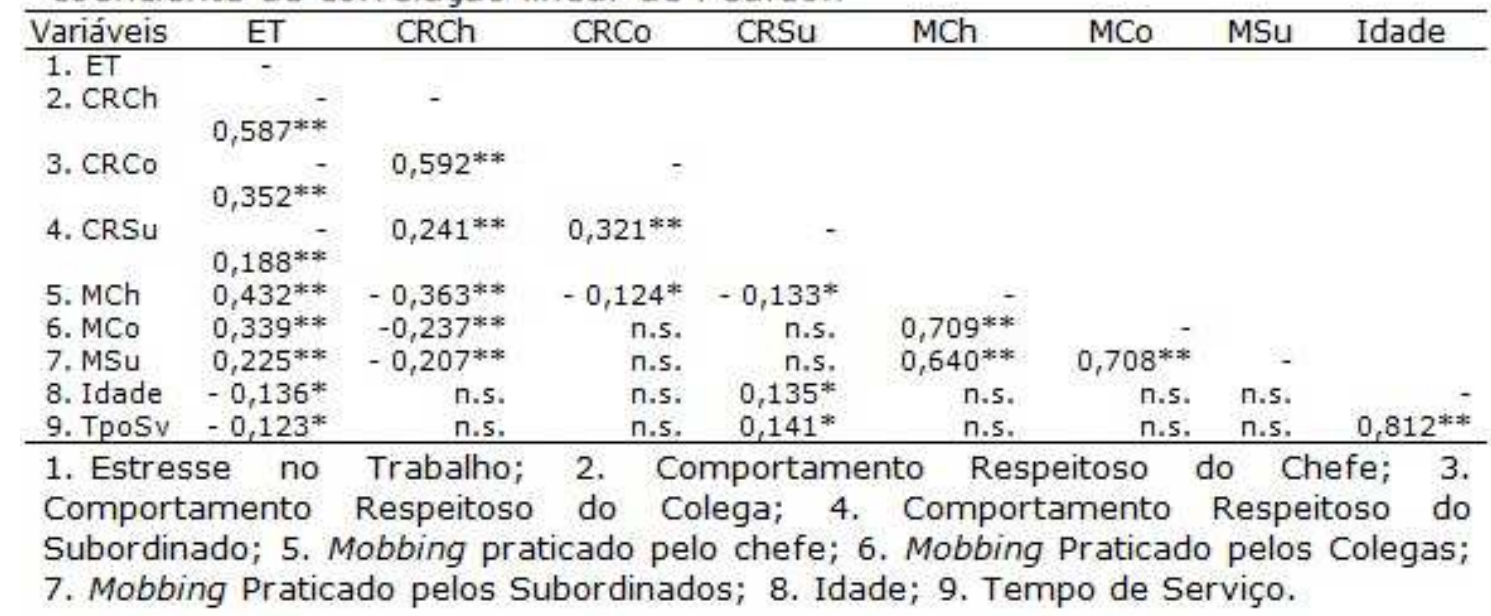

n.s. - Não Significativa; ${ }^{* *}$ - Significativa ao nivel de 0,$01 ; *$ - Significativa ao nível de 0,05 .

As correlações obtidas foram todas significativas e no sentido esperado. Assim, há uma correlação positiva significativa do estresse no trabalho com todos os casos de mobbing. O estresse se correlacionou negativamente como todos os casos de comportamento respeitoso no ambiente de trabalho.

O fato da idade e do tempo de serviço terem se correlacionado de forma negativa com o estresse no trabalho indicam um fenômeno típico da instituição investigada, em que há um respeito por aqueles servidores que são mais experientes ou mesmo mais velhos. Tal evidência aparece novamente ao se considerar a correlação positiva significativa que foi obtida na relação do comportamento respeitoso por parte do subordinado com idade e tempo de serviço.

\section{Conclusões}

O objetivo do presente estudo foi a validação da Escala de Mobbing no Trabalho. Diante dos resultados obtidos e dos valores satisfatórios indicados nos modelos testados, pode-se dizer que tal objetivo foi atingido.

De fato, a escala apresenta-se como uma alternativa para mensuração do mobbing em estudos brasileiros, fator importante considerando a escassez de instrumentos atualmente existente. Entretanto, é importante considerar que são três instrumentos independentes. O mobbing poderá então ser praticado por um dos três segmentos e não necessariamente pelos três ao mesmo tempo. 
Entretanto, chama a atenção o fato de terem sido obtidas correlações positivas significativas entre o mobbing praticado pelo chefe, pelos colegas e pelos subordinados. É possível que o indivíduo que é alvo de mobbing para um dos segmentos envolvidos (chefe, colegas ou subordinados), termine sendo para os outros seguimentos.

O aumento do número de estudos na área pode contribuir para uma discussão mais ampla do mobbing e de outras formas de opressão e discriminação no ambiente de trabalho, que muitas vezes são negligenciadas em investigações relacionadas a saúde e qualidade de vida no trabalho.

Os instrumentos propostos permitiram a obtenção de indicativos de validade externa dos instrumentos, ao serem obtidas relações entre mobbing e estresse no trabalho que ratificam as evidências que vêm sendo discutidas na literatura.

Estudos futuros poderiam ser realizados de modo a obter maiores indicativos da validade externa da escala, bem como testar sua estrutura em outros contextos. Sugere-se assim, buscar a inclusão de outras variáveis que vêm sendo indicadas na literatura em sua relação com o mobbing e estresse no trabalho.

Em organizações privadas é possível que novas informações possam ser obtidas, na relação entre mobbing e estresse no trabalho ou mesmo nos níveis de mobbing praticados por chefes, colegas e subordinados. Em relação ao mobbing praticado pelos subordinados, observa-se uma influência relativamente baixa, porém significativa, sobre o estresse no trabalho. Considerando os três tipos de mobbing do estudo, observa-se que o mobbing praticado pelo chefe foi maior, sendo seguido em grau de influência pelo praticado pelos companheiros de trabalho e, em último lugar, o mobbing praticado pelos subordinados. Estudos comparativos entre o setor público e 0 privado poderiam também permitir uma melhor compreensão sobre como a possibilidade de demissão influencia o mobbing, pincipalmente aquele praticado por subordinados.

O estudo contribui para uma melhor compreensão tanto do estresse no trabalho, quanto do próprio mobbing. Isso por que ao apontar o mobbing praticado por chefes, colegas e subordinados como mais um fator capaz de causar danos ao trabalhador, chama a atenção para um fenômeno presente no ambiente organizacional, mas que tem sido pouco identificado e negligenciado nos estudos sobre estresse no trabalho. Da mesma forma, ao evidenciar a influência do mobbing sobre 0 estresse abre a possibilidade para investigações que busquem identificar que outras variáveis podem ser influenciadas $n$ pelo mobbing no ambiente de trabalho. Os dados obtidos no presente estudo, entretanto, devem ser interpretados com precaução, sendo necessários mais estudos de modo a se obter maiores evidências da validade externa dos instrumentos. 


\section{Referências}

Baptista, M. N., Rueda, F. J. M., \& Sisto, F. F. (2007). Relação entre estresse laboral e atenção concentrada. Encontro: Revista de Psicologia, 11(16), 75-89.

Bernal, A. O. (2010). Psicologia do trabalho em um mundo globalizado: como enfrentar o assédio psicológico e o estresse no trabalho. Artmed: Porto Alegre.

Canova, K. R. \& Porto, J. B. (2010). O impacto dos valores organizacionais no estresse ocupacional de professores do ensino médio. Revista de Administração Mackenzie, 11(5), 431.

Cartwright, S. \& Whatmore, S. C. (2005). Stress and individual differences: implications for stress management. In: AlexanderStamatios G. Antoniou \& Cary L. Cooper (Eds.). Research Companion to Organizational Health Psychology. Massachussets: Edward Elgar Publishing, Inc. pp. 163-173.

Cox, T. (1993). Stress Research and Stress Management: Putting Theory to Work, Sudbury: HSE Books.

Einarsen, S. \& Hoel, H. (2001). The Negative Acts Questionnaire: Development, validation and revision of a measure of bullying at work. Paper presented at the 10th. European Congress on Work and Organisational Psychology, Prague.

Fidalgo, A. M. \& Piñuel, I. (2004). La escala Cisneros como herramienta de valoración del mobbing. Psicothema. 16 (4), 615-624.

Gonzalez de Rivera, J. L. \& Rodríguez-Abuin, M. J. (2005). Cuestionario de estrategias de acoso en el trabajo. EI LIPT-60. Madrid: Editorial EOS.

Guimarães, L. A. M. \& Rimoli, A. O. (2006). "Mobbing" (assédio psicológico) no trabalho: uma síndrome psicossocial multidimensional. Psicologia: Teoria e Pesquisa, 22(2), 183191.

Hair, J. F., Black, W. C., Babin, B. J., Anderson, R. E., \& Tatham, R. L. (2009). Análise multivariada de dados. Porto Alegre: Bookman.

Heaphy, E. D. \& Dutton, J. E. (2008). Positive social interactions and the human body at work: linking organizations and physiology. Academy of Management Review, 33(1), 137-162.

Jex, S. M. (2002). Organizational psychology: A scientist-practitioner approach. Nova York: John Wiley, Sons.

Jong, E. D. (2002). Well-being, Stress Management and Performance: From Analysis to Intervention. In: Sabine Sonnentag (Ed.) Psychological Management of Individual Performance. New York: John Wiley \& Sons, Ltd. pp. 425-440. 
Lee, R. \& Brotheridge, C. (2006). When prey turn predatory: Workplace bullying as a predictor of counter aggression/bullying, coping, and well being. European Journal of Work and Organizational Psychology, 15, 352-377.

Leymann, H., \& Tallgren, U. (1989). Undersokning av frekven-sen vuxenmobbning inom SSAB (Investigation into the frequency of adult mobbing in SSAB a Swedish steel company using the LIPT questionnaire). Arbete, miinniska, miljo, 1, lla-115. 1989.

Leymann, H. (1990). The mobbing encyclopaedya: The definition of mobbing at workplaces. 12100e. Recuperado em 24 abril, 2014 de http://leymann.se/English/frame.html

Marôco, J. (2010). Análise de equações estruturais: fundamentos teóricos, software e aplicações. Lisboa: Report Number Ltda.

Martinez, M. C. \& Latorre, M. R. D. O. (2009). Fatores associados à capacidade para o trabalho de trabalhadores do Setor Elétrico. Cadernos de Saúde Pública, 25(4), 761-772.

Namie, G., \& Namie, R. (1999). Bullyproof yourself at work! Personal strategies to stop the hurt from harassment. Benicia, CA: DoubleDoc Press.

Paschoal, T. \& Tamayo, A. (2005). Impacto dos valores laborais e da interferência Família-trabalho no estresse ocupacional. Psicologia: Teoria e Pesquisa, 21(2), 173-180.

Paschoal, T. \& Tamayo, A. (2004). Validação da Escala de estresse no Trabalho. Estudos de Psicologia, 9(1), 45-52.

Plassat, X. \& Gonçalves, J. B. A. (2005). Escravidão: paradigma do agronegócio? In: A. U. Oliveira \& J. P. Stedile (Orgs.). A natureza do agronegócio no Brasil. (pp. 45-50). Brasília: Via Campesina.

Sadir, M. A., Bignotto, M. M., \& Lipp, M. E. N. (2010). Stress e qualidade de vida: influência de algumas variáveis pessoais. Paidéia, 20(45), 73-81.

Santos, J. A. F. (2009). A interação estrutural entre a desigualdade de raça e de gênero no Brasil. Revista Brasileira de Ciências Sociais, 24(70), 37-60.

Selye, H. (1950). Stress and general Adaptation Syndrome. British Medical Journal, 17, 1383-1392.

Shallcros, L., Shehan, M., \& Ramsay, S. (2008). Workplace mobbing: experience in the public sector. International Journal of Organisational Behaviour, 13(2), 56-70.

Tamayo, A. (2001). Prioridades axiológicas, atividade física e estresse ocupacional. Revista de Administração Contemporânea, 5(3), 127-147.

Tang, J. L. (2008). A Study of Work Stress, Organizational Commitment, Job Satisfaction, and Organizational Citizenship Behavior: A Case to Employee Who are Taking Further 
Education in University. The Journal of Human Resource and Adult Learning, 4(1), 104-112.

Westhues, K. (2003). The mobbings at Medaille College in 2002. New York Academy, 30(1), 8-10.

White, B. (1994). Children, work and child labour: Changing responses to the employment of children. Development and Change, $25: 849-878$.

\section{Endereço para correspondência \\ Tássia Donadello Ferreira Lima}

Universidade Federal Rural do Rio de Janeiro

Departamento de Psicologia

BR 465, Km 7, Seropédica, CEP 23890-000, Rio de Janeiro - RJ, Brasil

Endereço eletrônico: tassiadf_psi@yahoo.com.br

\section{Marcos Aguiar de Souza}

Universidade Federal do Rio de Janeiro

Programa de Pós Graduação em Psicologia

Av. Pasteur, 250, Urca, CEP 22290-902, Rio de Janeiro - RJ, Brasil

Endereço eletrônico: marcos.aguiar@pq.cnpq.br

Recebido em: 05/11/2014

Reformulado em: 06/05/2015

Aceito para publicação em: 11/05/2015

\section{Notas}

* Graduação em Psicologia pela Universidade Veiga de Almeida (2007), PósGraduação Lato sensu em Psicologia Educacional (2010) pela Universidade Federal Rural do Rio de Janeiro e Mestrado em Psicologia pela Universidade Federal Rural do Rio de Janeiro (2014).

** Mestrado em Psicologia pela Universidade Gama Filho (1995), doutorado em Psicologia pela Universidade Federal do Rio de Janeiro (2003) e pós-doutorado em gestão, com ênfase em métodos quantitativos, no Instituto Superior de Ciências do Trabalho e da Empresa, do Instituto Universitário de Lisboa - ISCTE-IUL. 